\title{
Light scattering study of acoustic phonon modes and central peaks in $\mathrm{Pb}\left[\left(\mathrm{Mg}_{1 / 3} \mathrm{Nb}_{2 / 3}\right)_{0.45} \mathrm{Ti}_{0.55}\right] \mathrm{O}_{3}$ single crystals
}

\author{
Jae-Hyeon $\mathrm{Ko}^{\mathrm{a})}$ \\ Department of Physics, Hallym University, 39 Hallymdaehakgil, Chuncheon, Gangwondo 200-702, \\ Republic of Korea \\ Seiji Kojimab) \\ Institute of Materials Science, University of Tsukuba, Tsukuba City, Ibaraki 305-8573, Japan \\ Alexei A. Bokov and Zuo-Guang Ye ${ }^{\text {c) }}$ \\ Department of Chemistry, Simon Fraser University, Burnaby, British Columbia, V5A 1S6, Canada
}

(Received 23 October 2007; accepted 27 November 2007; published online 21 December 2007)

\begin{abstract}
The dynamic polar nanoregions (PNRs) which form below the Burns temperature and determine the unique properties of relaxor ferroelectrics were predicted [Tinte et al., Phys. Rev. Lett. 97, 137601 (2006)] to be pinned to the quenched chemically ordered regions (CORs) usually observed in lead-containing relaxors. In $\mathrm{Pb}\left[\left(\mathrm{Mg}_{1 / 3} \mathrm{Nb}_{2 / 3}\right)_{0.45} \mathrm{Ti}_{0.55}\right] \mathrm{O}_{3}$ crystal where CORs are known to be absent, we have found the phenomena typically related to the relaxation of dynamic PNRs, namely, the significant Brillouin quasielastic scattering, the softening of the longitudinal acoustic mode, and the deviation from the Curie-Weiss law above the Curie point. This implies that PNRs may appear in crystals without CORs. (C) 2007 American Institute of Physics. [DOI: 10.1063/1.2825416]
\end{abstract}

Recently, relaxor-based ferroelectric perovskite $\mathrm{Pb}\left[\left(\mathrm{Zn}_{1 / 3} \mathrm{Nb}_{2 / 3}\right)_{1-x} \mathrm{Ti}_{x}\right] \mathrm{O}_{3} \quad(\mathrm{PZN}-x \mathrm{PT}) \quad$ and $\mathrm{Pb}\left[\left(\mathrm{Mg}_{1 / 3} \mathrm{Nb}_{2 / 3}\right)_{1-x} \mathrm{Ti}_{x}\right] \mathrm{O}_{3}$ (PMN-xPT) single crystals have attracted great attention as promising materials in various electromechanical applications because of their superior piezoelectric properties. ${ }^{1}$ These complex compounds are formed by combining relaxor ferroelectrics (RFEs) and lead titanate $\left(\mathrm{PbTiO}_{3}\right)(\mathrm{PT})$ ferroelectric materials. RFEs have been studied intensively during the last two decades from the viewpoints of fundamental physics as well as industrial applications. ${ }^{2}$ RFEs exhibit unusual, complex properties different from those of normal ferroelectric materials. For example, $\mathrm{Pb}\left(\mathrm{Mg}_{1 / 3} \mathrm{Nb}_{2 / 3}\right) \mathrm{O}_{3}(\mathrm{PMN})$, an archetypal RFE, shows a diffuse, frequency-dependent broad dielectric maximum, broad distribution of relaxation times with divergent leading edge on cooling, no macroscopic symmetry change down to the lowest temperature, and the existence of nanosized polar regions (PNRs) below a certain temperature, the so-called Burns temperature $\left.\left(T_{B}\right)\right)^{3,4}$ Recent first-principles-based simulations of RFEs suggested that the PNRs may appear predominantly in the short-range chemically ordered regions (CORs). ${ }^{5}$

One of the key questions on PNRs of RFEs is about the upper limit of the PT content up to which the PNRs persist in PZN- $x$ PT and PMN- $x$ PT single crystals. Recent neutron study on PMN-60\%PT showed negligible diffuse scattering related to PNRs, suggesting that short-ranged polar correlations do not form in PMN-60\% $\mathrm{PT}^{6}$ In contrast, dielectric properties of PMN-55\%PT showed relaxorlike behavior, indicating the possibility of the existence of PNRs in this high-PT composition. ${ }^{7}$ In this previous study, it was found that the dielectric constant measured along the [100] direction follows the Curie-Weiss law (CW law) in the hightemperature range but deviates from it at about $610 \mathrm{~K}$, which

\footnotetext{
${ }^{a)}$ Electronic mail: hwangko@hallym.ac.kr.

${ }^{b)}$ Electronic mail: kojima@bk.tsukuba.ac.jp.

${ }^{\text {c) }}$ Electronic mail: zye@sfu.ca.
}

will be denoted as $T_{B}$ for convenience. Furthermore, the temperature dependence of the dielectric constant in the temperature range from the ferroelectric transition temperature $\left(T_{C}=521 \mathrm{~K}\right)$ to $T_{B}$ could be described by an empirical quadratic scaling relation. ${ }^{8}$ These results strongly indicate some relaxorlike nature in this $\mathrm{PMN}-x \mathrm{PT}$ of high-PT content. This letter reports on detailed Brillouin light scattering study on PMN-55\% PT single crystals in a wide temperature range, which shows very strong relaxational dynamics as well as significant softening of acoustic modes above $T_{C}$.

The $\mathrm{Pb}\left[\left(\mathrm{Mg}_{1 / 3} \mathrm{Nb}_{2 / 3}\right)_{1-x} \mathrm{Ti}_{x}\right] \mathrm{O}_{3}$ single crystals were grown by the Bridgman method with nominal composition $x=0.5$ and cut to obtain (100)-oriented platelets. ${ }^{7}$ It is known that the real composition of these solid solution crystals is usually different from the nominal composition. By comparing the Curie point $T_{C}$ of the crystals with the published $T-x$ phase diagram, ${ }^{6}$ we found that $x \cong 0.55 . T_{C}$ turns out to be equal to the maximum dielectric constant temperature $\left(T_{m}\right)$. A diode pumped solid state laser at the wavelength of $532 \mathrm{~nm}$ was used as an excitation light source. A microBrillouin scattering system with a $3+3$ pass Sandercock tandem Fabry-Pérot interferometer was used to measure the Brillouin spectra at a backward scattering geometry.

In the cubic phase of solids, only the longitudinal acoustic (LA) mode can be observed from the Brillouin spectrum at a backward scattering geometry. The appearance of the transverse acoustic (TA) mode at this geometry, thus, indicates a structural phase transition from cubic to tetragonal symmetry. The PMN-55\%PT undergoes a ferroelectric phase transition at $T_{C}=521 \mathrm{~K}$, above which only one Brillouin doublet from LA mode could be seen in this study. At and below $T_{C}$, additional Brillouin doublet corresponding to the TA mode was clearly observed. The inset figure of Fig. 1(b) shows one example of the Brillouin spectra measured at $507 \mathrm{~K}<T_{C}$, which shows two acoustic modes as well as a CP. Figure 1(a) shows the fitted result for the Brillouin frequency shifts of the LA and TA modes (denoted as $\nu_{\mathrm{LA}}$ and $\nu_{\mathrm{TA}}$, respectively) as a function of temperature. Figure 1(b) 


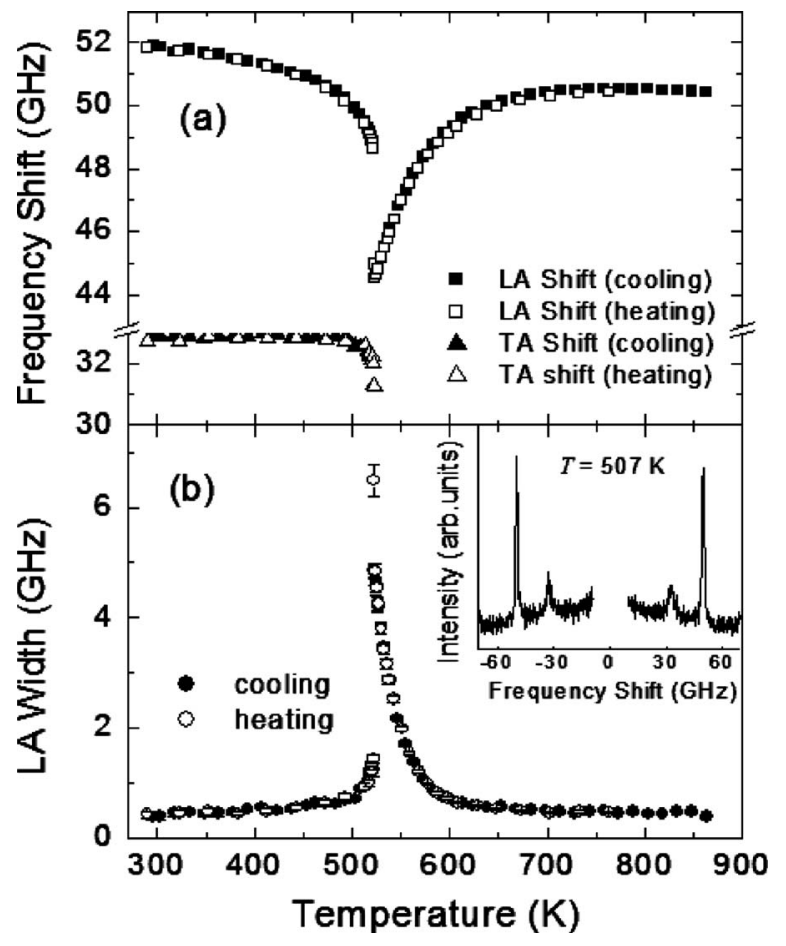

FIG. 1. (a) Temperature dependence of the Brillouin shift of LA and TA modes and (b) the FWHM of the LA mode measured at both cooling and subsequent heating processes. Inset: A Brillouin spectrum measured at $507 \mathrm{~K}$.

shows the full width at half maximum (FWHM) of the LA mode, which is related to the acoustic damping of this mode. $\nu_{\mathrm{LA}}$ shows a very mild change at above $\sim 600 \mathrm{~K}$, indicating that only normal anharmonic effects dominate the lattice dynamics in this high-temperature range. Below $600 \mathrm{~K}, \nu_{\mathrm{LA}}$ shows a substantial softening upon approaching $T_{C}$. In the same temperature range, the FWHM exhibits a sharp increase, suggesting a significant coupling between the LA waves and other degrees of freedom. When the crystal undergoes ferroelectric phase transition at $521 \mathrm{~K}$ upon cooling, $\nu_{\mathrm{LA}}$ shows a discontinuous change to a higher value concomitant with the appearance of the TA mode, which indicates a structural phase transition from cubic to tetragonal symmetry.

Figure 2(a) shows the change of the Brillouin spectra as a function of temperature recorded at three selected temperatures in the range of $T_{C}-T_{B}$. It is readily recognizable that $\mathrm{CP}$ appears at $\sim 570 \mathrm{~K}$ and grows substantially upon cooling. The intensity of the polarized component of $\mathrm{CP}$ was much larger than the depolarized one, suggesting that the $\mathrm{CP}$ is strongly polarized. The $\mathrm{CP}$ was nicely fitted by using a single Lorentzian function centered at zero frequency when assuming a Debye relaxation process. The temperature dependence of the integrated intensity of the $\mathrm{CP}$ component is plotted in Fig. 2(b). The inverse of the dielectric permittivity $(\epsilon)$ is also shown in the same figure for comparison. The solid line is a fitted result to the $\mathrm{CW}$ law of $1 / \epsilon=\left(T-T_{\mathrm{CW}}\right) / C$. $\epsilon$ follows the $\mathrm{CW}$ law in the high-temperature range with $T_{\mathrm{CW}}=531 \mathrm{~K}$ and $C=1.7 \times 10^{5}$, but it shows a deviation from the high-temperature linearity at $T_{B} \sim 610 \mathrm{~K}$. This deviation becomes more noticeable at about $570 \mathrm{~K}$, at which $\mathrm{CP}$ begins to appear and grow significantly on lowering the temperature. The intensity of $\mathrm{CP}$ reaches a maximum exactly at $T_{C}$.

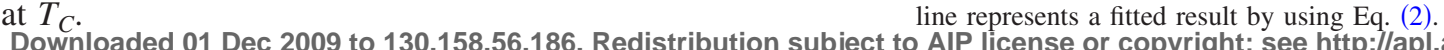
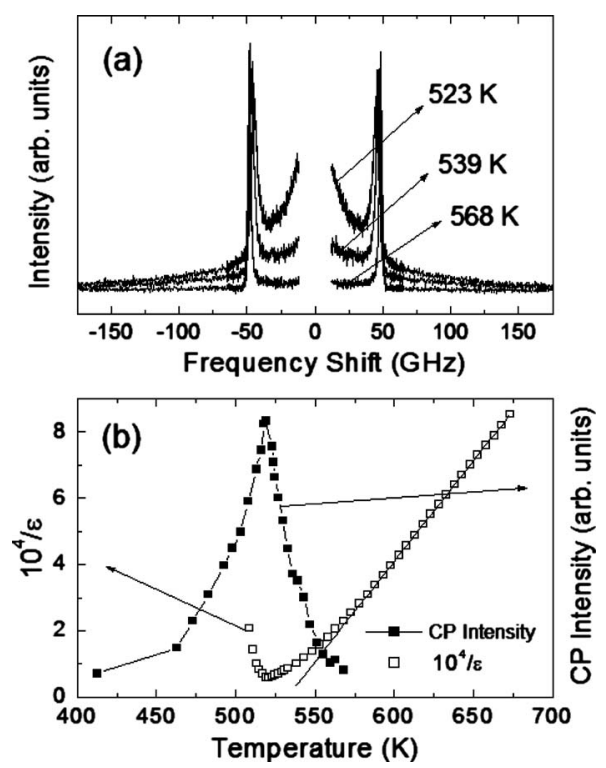

FIG. 2. (a) Brillouin spectra measured at three selected temperatures. (b) The temperature dependence of the inverse dielectric permittivity (solid squares) and the intensity of $\mathrm{CP}$ (open squares). The solid line is a fitted result to the $\mathrm{CW}$ law.

It has been found that the dielectric constant of various relaxor samples from the high-temperature slopes can be explained by using the following empirical equation: ${ }^{8}$

$$
\frac{\epsilon_{A}}{\epsilon}=1+\frac{\left(T-T_{A}\right)^{2}}{2 \delta^{2}}
$$

where $T_{A}$ and $\epsilon_{A}$ are parameters used for defining the temperature position of the peak and the extrapolated value of the dielectric constant at $T=T_{A}$, and $\delta$ is a shape parameter describing the diffuseness of the dielectric properties of relaxors. Figure 3(a) gives the fitting result for the real part of the permittivity of the PMN-55\%PT, which shows that the quadratic law of Eq. (1) is fulfilled in the temperature range of $521-580 \mathrm{~K}$ with fitting parameters of $\epsilon_{A}=3.6 \times 10^{4}$,

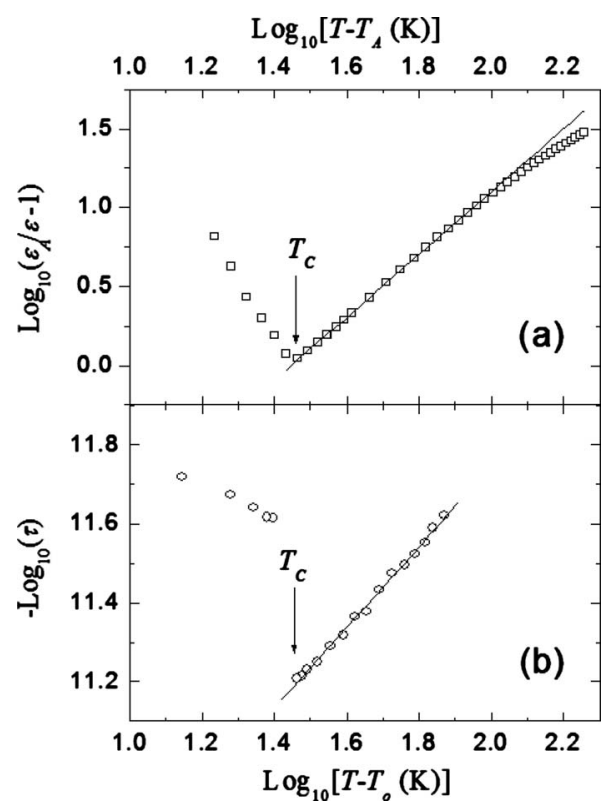

FIG. 3. (a) The temperature dependence of the dielectric permittivity fitted by Eq. (1). The solid line represents a fitted result. (b) The temperature dependence of the relaxation time estimated from the width of CP. The solid 
$T_{A}=492 \mathrm{~K}$, and $\delta=20 \mathrm{~K}$. These results suggest that the dielectric properties of PMN-55\% PT between $T_{C}$ and $T_{B}$ are characterized by typical relaxor behavior rather than by the $\mathrm{CW}$ law commonly observed in normal ferroelectric materials, despite the fact that it undergoes a first-order ferroelectric phase transition at $T_{C}$. This unusual behavior is correlated with the strong growth of the $\mathrm{CP}$ component in light scattering spectrum. The half width of CP is inversely proportional to the relaxation time $\tau$ of the related polarization fluctuations. ${ }^{9}$ The estimated relaxation time increases substantially on cooling from $570 \mathrm{~K}$ towards $T_{C}$, which indicates slowing down of the relevant dynamics in the hightemperature phase $\left(T_{C}<T<T_{B}\right)$ of PMN-55\% PT. Therefore, we apply the following well-known relationship between $\tau$ and the temperature to fit the data: ${ }^{10}$

$$
\frac{1}{\tau}=\frac{T-T_{0}}{\tau_{0} T_{0}} .
$$

Figure 3(b) shows the fitting result for the relaxation times above $T_{C}$, which results in $\tau_{0}=0.36 \mathrm{ps}$ and $T_{0}=494 \mathrm{~K}$. Therefore, Eq. (2) nicely explains the temperature dependence of $\tau$ in the high-temperature phase of PMN-55\%PT. This observation suggests that the $\mathrm{CP}$ originates from the polarization fluctuations and that an order-disorder mechanism contributes to the slowing down of the polarization fluctuations in PMN-55\%PT.

However, the phase transition behavior of PMN-55\%PT should be differentiated from the critical behavior observed in PT, as well as in typical order-disorder-type ferroelectrics, because the dielectric constant of PMN-55\%PT does not diverge according to the $\mathrm{CW}$ law. Instead, a strong relaxational mode whose polarization fluctuations display a slowing down is accompanied by relaxorlike dielectric behavior in the high-temperature phase between $T_{C}$ and $T_{B}$. Considering the existence of a peculiar temperature $T_{B}$ below which the dielectric permittivity deviates from the $\mathrm{CW}$ law, the most probable origin of the strong central peak and significant softening of the LA mode observed in PMN-55\%PT is the presence of PNRs and their relaxational dynamics. Note that the strong spatial correlation between PNRs and nanoscale regions of chemical ordering (or CORs) on the $B$ sites was suggested by recent first-principles-based simulations. ${ }^{5}$ However, in PMN- $x$ PT solid solutions, the size of COR decreases with increasing $x$ and they completely disappear at $x$ exceeding $\sim 0.4$. This was found by transmission electron microscopy ${ }^{11}$ and confirmed by synchrotron x-ray scattering studies. ${ }^{12}$ Therefore, the PNRs we observed in PMN-55\%PT crystal are not related to the CORs. This implies that the existence of CORs is not a necessary condition for the formation of PNRs: the latter may nucleate at $T<T_{B}$ in the chemically disordered matrix.

However, Stock et al. reported from their neutron scattering and $\mathrm{x}$-ray diffraction experiments on $\mathrm{PMN}-60 \% \mathrm{PT}$ that the diffuse scattering was found to be weak and temperature independent and, thereby, it was concluded that PNRs are not present in PMN-60\% PT crystals (close to the composition of our crystal). ${ }^{6}$ To explain this apparent discrepancy with our results, we note that, according to the high energy resolution neutron spin-echo spectroscopy study, the diffuse scattering (in PMN) is purely elastic (within an experimental accuracy of $1 \mu \mathrm{eV}$ ) and, therefore, can be associated with static (or quasistatic) PNRs [whose relaxation rate is less than $250 \mathrm{MHz}(1 \mu \mathrm{eV})]^{13}$ It was further sug- gested that the static PNRs are formed inside the CORs, while the dynamic relaxing PNRs appear in the chemically disordered matrix. ${ }^{14}$ The dynamic PNRs presumably can be observed only at very small scattering vectors inaccessible in the neutron and x-ray experiments. ${ }^{13}$ In accordance with these ideas, neutron and $\mathrm{x}$-ray diffuse scattering should not be observed in PMN- $x$ PT crystals with $x \sim 0.5$, where CORs (and thereby static PNRs) are absent. On the other hand, by means of quasielastic light scattering (i.e., working with small wave vectors), we have found in PMN-55\%PT crystal a significant central peak which, thereby, can be related to the dynamic PNRs. The relaxation of these dynamic PNRs give rise to the dielectric peak described by relation (1), which is typical of relaxor behavior.

To summarize, it was found that the phase transition of PMN-55\% PT is characterized by two characteristic temperatures, $T_{B} \sim 610 \mathrm{~K}$ and $T_{C}=521 \mathrm{~K}$. Above $T_{B}$, the dielectric permittivity $\epsilon$ follows the $\mathrm{CW}$ law and there is no substantial change in acoustic properties. Between $T_{B}$ and $T_{C}, \epsilon$ deviates from the CW law and follows the quadratic scaling relationship similar to other typical relaxors, and a strong central peak grows upon cooling accompanied by substantial softening of the LA mode. At $T_{C}$, the Brillouin shift of the LA mode shows a discontinuous change in addition to the appearance of the TA mode, indicating a typical structural phase transition. All these results indicate that dynamic PNRs still persist even in PMN-55\%PT without CORs, which is responsible for the relaxorlike behavior observed in the high-temperature phase $\left(T_{C}<T<T_{B}\right)$, even though a long-range ferroelectric phase is achieved due to the weakened random fields and the enhanced anisotropy with increased PT content.

The authors are thankful to S. Tsukada of University of Tsukuba for his assistance in carrying out the experiment. This work was supported by the Korea Research Foundation Grant funded by the Korean Government (MOEHRD, Basic Research Promotion Fund) (No. KRF-2006-331-C00088) and by the U. S. Office of Naval Research (Grant No. N00014-06-1-0166).

${ }^{1}$ S.-E. Park and T. R. Shrout, J. Appl. Phys. 82, 1804 (1997).

${ }^{2}$ A. A. Bokov and Z.-G. Ye, J. Mater. Sci. 41, 313 (2006).

${ }^{3}$ L. E. Cross, Ferroelectrics 76, 241 (1987).

${ }^{4}$ G. Burns and F. H. Dacol, Ferroelectrics 104, 25 (1990).

${ }^{5}$ S. Tinte, B. P. Burton, E. Cockayne, and U. V. Waghmare, Phys. Rev. Lett. 97, 137601 (2006).

${ }^{6}$ C. Stock, D. Ellis, I. P. Swainson, G. Xu, H. Hiraka, Z. Zhong, H. Luo, X. Zhao, D. Viehland, R. J. Birgeneau, and G. Shirane, Phys. Rev. B 73, 064107 (2006)

${ }^{7}$ A. A. Bokov, H. Luo, and Z.-G. Ye, Mater. Sci. Eng., B 120, 206 (2005).

${ }^{8}$ A. A. Bokov, Y.-H. Bing, W. Chen, Z.-G. Ye, S. A. Bogatina, I. P. Raevski, S. I. Raevskaya, and E. V. Shakar, Phys. Rev. B 68, 052102 (2003).

${ }^{9}$ J.-H. Ko, D. H. Kim, and S. Kojima, J. Korean Phys. Soc. 49, S536 (2006).

${ }^{10}$ M. E. Lines and A. M. Glass, Priciples and Applications of Ferroelectrics and Related Materials, (Clarendon, Oxford, 1977), p. 50.

${ }^{11}$ A. D. Hilton, D. J. Barber, C. A. Randall, and T. R. Shrout, J. Mater. Sci. 25, 3461 (1990)

${ }^{12}$ A. Tkachuk, P. Zschack, E. Colla, and H. Chen, Fundamental Physics of Ferroelectrics (American Institute of Physics, New York, 2001), vol. 582, p. 45.

${ }^{13}$ S. Vakhrushev, A. Ivanov, and J. Kulda, Phys. Chem. Chem. Phys. 7, 2340 (2005).

${ }^{14} \mathrm{~S}$. Vakhrushev, Abstract Book of the 11th European Meeting on Ferroelectricity, Bled, Slovenia, 2007 (unpublished) p. 219. 